\title{
The Magnetic Field Produced by the Heart and Its Influence on MRI
}

\author{
Dan Xu and Bradley J. Roth \\ Department of Physics, Oakland University, Rochester, MI, USA \\ Correspondence should be addressed to Bradley J. Roth; roth@oakland.edu
}

Received 5 February 2017; Accepted 20 April 2017; Published 10 May 2017

Academic Editor: Seungik Baek

Copyright (C) 2017 Dan Xu and Bradley J. Roth. This is an open access article distributed under the Creative Commons Attribution License, which permits unrestricted use, distribution, and reproduction in any medium, provided the original work is properly cited.

\begin{abstract}
Background. Action currents in the heart produce a magnetic field, which could provide a way to detect the propagation of electrical activity through cardiac tissue using magnetic resonance imaging. However, the magnetic field produced by current in the heart is small. The key question addressed in this study is are cardiac biomagnetic fields large enough to be detectable by MRI? Results. A spherical model is used to calculate the magnetic field inside the heart, which has a magnitude of about $14 \mathrm{nT}$. This field implies a phase shift in the MRI signal of about $0.2^{\circ}$. Conclusion. Phase shifts associated with cardiac action currents will be difficult to detect using current MRI technology but may be possible if motion artifacts and other physiological noise can be suppressed.
\end{abstract}

\section{Introduction}

Action currents in the heart produce a magnetic field, which could create an artifact in a magnetic resonance image (MRI). Viewed from another perspective, in theory MRI could be used to image action currents by detecting their magnetic field. Indeed, if one could image action currents using MRI, it would be a fundamental advance that might make magnetocardiography and even much of electrocardiography obsolete. However, the magnetic field produced by current in the heart is small. The key question addressed in this study is are biomagnetic fields large enough to be detectable by MRI? The main goal of this paper is to compute how large this magnetic field is and explore the possibility of detecting the magnetic field by magnetic resonance imaging.

Over the last ten years, much work has been done to detect neuronal activation by measuring the magnetic field produced by biocurrents using MRI [1-8]. In general, these magnetic fields are so tiny that they are difficult to measure [3]. However, the heart is the source of the largest biocurrents in the body, so it may be easier to detect cardiac action currents using MRI compared to neural action currents [9]. In order to interpret and predict the MRI signal produced by cardiac action currents, we must know the magnetic field distribution within the heart.
In general, the best starting place for calculating the magnetic field is a simple model that clearly illustrates mechanisms. In this study, we examine a "spherical heart" model [10] with a simple transmembrane potential distribution and calculate the resulting action currents and magnetic field. A simple model allows us to obtain analytical solutions that provide insight into the qualitative behavior and allows us to estimate the quantitative magnitude of the magnetic field.

There are two sources that produce a magnetic field in cardiac tissue: one is the intracellular current in the tissue with the "return" loops through an adjacent volume conductor, and the other is the anisotropy of the tissue [11]. In this study we account for the first of these mechanisms: the heart is surrounded by a volume conductor that provides a path for return currents and results in a magnetic field.

\section{Methods}

We consider a spherical shell of cardiac tissue surrounding a blood cavity and surrounded by an unbounded conducting bath [10] (Figure 1). We use spherical coordinates $(r, \theta$, and $\phi)$ to specify position. The inner and outer radii of the shell are $r_{1}$ and $r_{2}$, respectively. The blood (subscript " $b$ ") conductivity is $g_{b}$ and the bath (subscript " $o$ ") conductivity is $g_{o}$. 


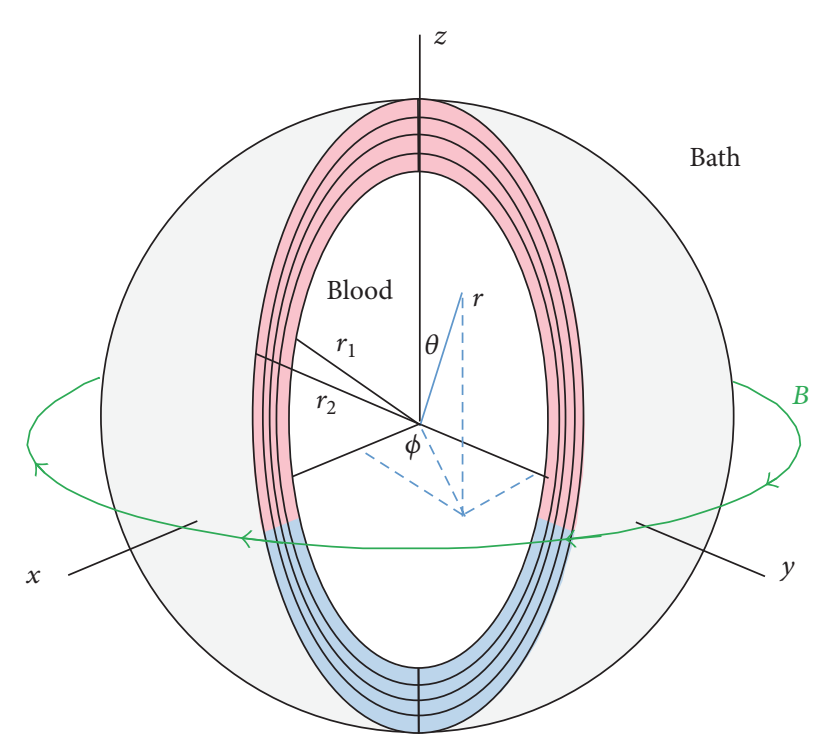

FIgURE 1: The model of a spherical heart. Part of the spherical shell has been cut out to show the heart wall. The black curves indicate the fiber orientation. The pink tissue has a transmembrane potential of $20 \mathrm{mV}$, and the blue tissue has a transmembrane potential of $-80 \mathrm{mV}$. The green curve shows the magnetic field. The endocardial (inner) surface has radius $r_{1}$, and the epicardial (outer) surface has radius $r_{2}$.

We assume the transmembrane potential has azimuthally symmetry. Therefore, it does not depend on the angle $\phi$ around the heart. With an analogy to earth, the transmembrane potential only depends on latitude but not longitude. The fiber geometry in the heart is complex, and we must simplify it significantly in order to obtain an analytical solution. Here the fiber axis is parallel to the meridians, that is, along lines of constant longitude (lie along $\theta$ direction) [10]. With this fiber model, we can calculate analytically the most significant information that we want, that is, the magnitude of the magnetic field $\mathbf{B}$.

We use the bidomain model to represent the electrical properties of cardiac tissue, which accounts for anisotropy both inside and outside the myocardial cells [12]. The bidomain model is a continuum model, in the sense that it represents the electrical properties of the tissue averaged over many cells [13]. The electrical conductivities in cardiac tissue $\left(g_{i r}, g_{i \theta}, g_{e r}\right.$, and $\left.g_{e \theta}\right)$ are different in $r$ and $\theta$ directions (perpendicular and parallel to the fibers) and in the intracellular (subscript " $i$ ") and extracellular (subscript " $e$ ") spaces.

We assume the tissue is quasistatic: the governing equations do not depend on time. The transmembrane potential will depend on time as the action potential propagates through the tissue, but, given the transmembrane potential at any moment, the other potentials, currents, and magnetic field can be calculated at that instant quasistatically.

2.1. Calculation of the Electrical Potential. In the cardiac shell, $r_{1}<r<r_{2}$, there are two potential fields, $V_{i}$ and $V_{e}$, which are functions of $r$ and $\theta$. The transmembrane potential is the difference of the intracellular and extracellular potentials:

$$
V_{m}=V_{i}-V_{e} .
$$

The fundamental equation governing the current density $\mathbf{J}$ is the equation of continuity

$$
\begin{array}{rl}
\nabla \cdot \mathbf{J}_{b}=0 & r<r_{1} \\
\nabla \cdot\left(\mathbf{J}_{i}+\mathbf{J}_{e}\right)=0 & r_{1}<r<r_{2} \\
\nabla \cdot \mathbf{J}_{o}=0 & r>r_{2} .
\end{array}
$$

Since we only require the sum of $\mathbf{J}_{i}$ and $\mathbf{J}_{e}$ be divergenceless, $\mathbf{J}_{i}$ and $\mathbf{J}_{e}$ individually need not have vanishing divergence. Thus, current can flow from one domain to another.

Using Ohm's law $\mathbf{J}=\tilde{g} \mathbf{E}$ and the relationship between the potential and the electrical field $\mathbf{E}=-\nabla V$, we find that $\mathbf{J}_{\mathbf{i}}=-\widetilde{g}_{i} \nabla V_{i}, \mathbf{J}_{\mathbf{e}}=-\widetilde{g}_{e} \nabla V_{e}, \mathbf{J}_{\mathbf{b}}=-g_{b} \nabla V_{b}$, and $\mathbf{J}_{o}=-g_{o} \nabla V_{o}$, with $\widetilde{g}_{i}=\left(\begin{array}{cc}g_{i r} & 0 \\ 0 & g_{i \theta}\end{array}\right)$ and $\widetilde{g}_{e}=\left(\begin{array}{cc}g_{e r} & 0 \\ 0 & g_{e \theta}\end{array}\right)$. If we use the above four current density expressions, then (2)-(4) become

$$
\begin{aligned}
& \nabla^{2} V_{b}=0 \\
& \frac{1}{r^{2}} \frac{\partial}{\partial r}\left(r^{2} \frac{\partial}{\partial r}\left(g_{i r} V_{i}+g_{e r} V_{e}\right)\right) \\
&+\frac{1}{r^{2} \sin \theta} \frac{\partial}{\partial \theta}\left(\sin \theta \frac{\partial}{\partial \theta}\left(g_{i \theta} V_{i}+g_{e \theta} V_{e}\right)\right)=0 \\
& \nabla^{2} V_{o}=0 .
\end{aligned}
$$

In order to simplify (6), we do a linear transformation from $V_{i}$ and $V_{e}$ to $V_{m}$ and $\psi[10,14]$ :

$$
\begin{aligned}
V_{m} & =V_{i}-V_{e}, \\
\psi & =V_{i}+\frac{g_{e \theta}}{g_{i \theta}} V_{e},
\end{aligned}
$$

with the inverse transformation given by

$$
\begin{aligned}
& V_{i}=\frac{g_{i \theta}}{g_{i \theta}+g_{e \theta}}\left(\psi+\frac{g_{e \theta}}{g_{i \theta}} V_{m}\right) \\
& V_{e}=\frac{g_{i \theta}}{g_{i \theta}+g_{e \theta}}\left(\psi-V_{m}\right) .
\end{aligned}
$$

If we substitute (9) and (10) into the expressions in (6), we get

$$
\begin{aligned}
& g_{i r} V_{i}+g_{e r} V_{e} \\
& \quad=\frac{g_{i \theta}}{g_{i \theta}+g_{e \theta}}\left\lfloor\left(g_{i r}+g_{e r}\right) \psi-g_{i r}\left(1-\frac{g_{i r} g_{e \theta}}{g_{e r} g_{i \theta}}\right) V_{m}\right\rfloor \\
& g_{i \theta} V_{i}+g_{e \theta} V_{e}=g_{i \theta} \psi .
\end{aligned}
$$

If we make the additional assumption that $V_{m}$ is not a function of $r$, the second term in the square brackets of (11) does not contribute to (6), and we obtain

$$
\frac{1}{\lambda^{2}} \frac{1}{r^{2}} \frac{\partial}{\partial r}\left(r^{2} \frac{\partial \psi}{\partial r}\right)+\frac{1}{r^{2} \sin \theta} \frac{\partial}{\partial \theta}\left(\sin \theta \frac{\partial \psi}{\partial \theta}\right)=0
$$


where

$$
\lambda=\sqrt{\frac{g_{i \theta}+g_{e \theta}}{g_{i r}+g_{e r}}} .
$$

2.2. Boundary Conditions. The behavior at the interface between cardiac tissue and an adjacent volume conductor is determined by boundary conditions $[15,16]$. At the surfaces of the shell, $r=r_{1}$ and $r=r_{2}$, we require that the normal component of the current density is continuous:

$$
\begin{array}{ll}
J_{i r}+J_{e r}=J_{b r} & r=r_{1} \\
J_{i r}+J_{e r}=J_{o r} & r=r_{2}
\end{array}
$$

and the extracellular potential is continuous

$$
\begin{array}{ll}
V_{e}=V_{b} & r=r_{1} \\
V_{e}=V_{o} & r=r_{2} .
\end{array}
$$

The boundary conditions in (15)-(17) give at $r=r_{1}$

$$
\begin{aligned}
\frac{\partial \psi}{\partial r} & =\lambda^{2} \frac{g_{b}}{g_{i \theta}} \frac{\partial V_{b}}{\partial r} \\
\psi & =V_{m}+\frac{g_{i \theta}+g_{e \theta}}{g_{i \theta}} V_{b} .
\end{aligned}
$$

For the boundary $r=r_{2}$, we replace $V_{b}$ by $V_{o}$ in (18).

2.3. Solution of the Equations for the Potential. If we expand $\psi$ in terms of Legendre polynomials [17], $P_{l}(\cos \theta)$, where $l=$ $0,1,2,3, \ldots$, then the general solution to (13) is [10]

$$
\psi=\sum\left(A_{l} r^{\nu_{1}}+B_{l} r^{\nu_{2}}\right) P_{l}(\cos \theta),
$$

where

$$
\begin{aligned}
\nu & =\sqrt{\lambda^{2} l(l+1)+\frac{1}{4}}, \\
v_{1} & =v-\frac{1}{2}, \\
v_{2} & =-v-\frac{1}{2} .
\end{aligned}
$$

The general solutions to Laplace's equation in the blood and bath are

$$
\begin{aligned}
& V_{b}=\sum_{l} C_{l} r^{l} P_{l}(\cos \theta), \\
& V_{o}=\sum_{l} D_{l} r^{-(l+1)} P_{l}(\cos \theta) .
\end{aligned}
$$

The boundary conditions determine the constants $A_{l}, B_{l}$, $C_{l}$, and $D_{l}$ for each $l$. We expand $V_{m}$ in terms of Legendre polynomials

$$
V_{m}=\sum_{l} F_{l} P_{l}(\cos \theta),
$$

where

$$
F_{l}=\frac{2 l+1}{2} \int_{0}^{\pi} V_{m}(\theta) P_{l}(\cos \theta) \sin \theta d \theta .
$$

Solving the four linear equations from the boundary conditions, we obtain the constants $A_{l}, B_{l}, C_{l}$, and $D_{l}$ for each $l$ :

$$
\begin{aligned}
A_{l} & =\frac{-b_{1}+b_{2}}{b_{2} a_{1}-a_{2} b_{1}} F_{l}, \\
B_{l} & =\frac{a_{1}-a_{2}}{b_{2} a_{1}-a_{2} b_{1}} F_{l}, \\
C_{l} & =\frac{A_{l} \nu_{1} r_{1}{ }^{\nu_{1}}+B_{l} \nu_{2} r_{1}{ }^{\nu_{2}}}{\lambda^{2}\left(g_{b} / g_{i \theta}\right) l r_{1}{ }^{l}}, \\
D_{l} & =-\frac{A_{l} \nu_{1} r_{2}{ }^{\nu_{1}}+B_{l} \nu_{2} r_{2}{ }^{\nu_{2}}}{\lambda^{2}\left(g_{o} / g_{i \theta}\right)(l+1) r_{2}{ }^{-(l+1)}},
\end{aligned}
$$

where

$$
\begin{aligned}
& a_{1}=r_{1}{ }^{\nu_{1}}\left(1-\frac{g_{i r}+g_{e r}}{g_{b}} \frac{v_{1}}{l}\right), \\
& b_{1}=r_{1}{ }^{\nu_{2}}\left(1-\frac{g_{i r}+g_{e r}}{g_{b}} \frac{v_{2}}{l}\right), \\
& a_{2}=r_{2}{ }^{\nu_{1}}\left(1+\frac{g_{i r}+g_{e r}}{g_{o}} \frac{v_{1}}{l+1}\right), \\
& b_{2}=r_{2}{ }^{\nu_{2}}\left(1+\frac{g_{i r}+g_{e r}}{g_{o}} \frac{v_{2}}{l+1}\right) .
\end{aligned}
$$

2.4. Calculation of the Magnetic Field. Using Ohm's law, we obtain the current density distribution

$$
\begin{aligned}
J_{b \theta} & =-\frac{1}{r} g_{b} \frac{\partial V_{b}}{\partial \theta} \quad r<r_{1}, \\
J_{\theta} & =J_{i \theta}+J_{e \theta}=-\frac{1}{r} g_{i \theta} \frac{\partial \psi}{\partial \theta} \quad r_{1}<r<r_{2}, \\
J_{o \theta} & =-\frac{1}{r} g_{o} \frac{\partial V_{o}}{\partial \theta} \quad r>r_{2} .
\end{aligned}
$$

Ampere's law in its integral form is

$$
\oint \overrightarrow{\mathbf{B}} \cdot d \mathbf{s}=\mu_{0} \iint \overrightarrow{\mathbf{J}} \cdot d \mathbf{S}=\mu_{0} I_{\text {enclosed }},
$$

where $I_{\text {enclosed }}$ is the current enclosed by the Amperian loop. We choose this loop to be the edge of a right circular cone with the apex at the center of the heart sphere and the integral surface is the surface of the right circular cone:

$$
\begin{aligned}
\overrightarrow{\mathbf{J}} & =-J \widehat{\mathbf{z}}=-J(\cos \theta \widehat{\mathbf{r}}-\sin \theta \widehat{\boldsymbol{\theta}}) \\
d \mathbf{S} & =-r \sin \theta d r d \phi \widehat{\boldsymbol{\theta}} .
\end{aligned}
$$

The magnetic field is in $\phi$ direction, tangential to this loop. By varying $r$ and $\theta$ of this cone, we can get the magnetic field in all the space

$$
B=\frac{\mu_{\mathrm{o}} I_{\mathrm{enclosed}}}{2 \pi r \sin \theta}
$$


where

$$
I_{\text {enclosed }}=-\int_{0}^{2 \pi} \int_{0}^{r} J_{\theta} r \sin \theta d r d \phi .
$$

In order to compute $I_{\text {enclosed }}$ and thereby the magnitude of $\mathbf{B}$, we need to substitute the potential ((19), (21), and (22)) into the expression of current density (30), and then replace $J_{\theta}$ in (34) by these expressions of current density in each region $r<r_{1}, r_{1}<r<r_{2}$, and $r>r_{2}$, respectively. We then sum up the integrals in a piecewise manner for the three spaces in blood, bath, and tissue.

In the blood $r<r_{1}$,

$$
\begin{aligned}
V_{b} & =\sum_{l} C_{l} r^{l} P_{l}(\cos \theta), \\
J_{\theta} & =J_{b \theta}=-\frac{1}{r} g_{b} \frac{\partial V_{b}}{\partial \theta}=-g_{b} \sum_{l} r^{l-1} C_{l} P_{l}^{1}, \\
I_{\text {enclosed }} & =-\int_{0}^{2 \pi} \int_{0}^{r} J_{\theta} r \sin \theta d r d \phi \\
& =g_{b} \sum_{l} C_{l} P_{l}^{1} 2 \pi \sin \theta \int_{0}^{r} r^{l} d r \\
& =g_{b} \sum_{l} C_{l} P_{l}^{1} 2 \pi \sin \theta \frac{r^{l+1}}{l+1}, \\
B_{\phi} & =\frac{\mu_{0}}{2 \pi r \sin \theta} I_{\text {enclosed }}=\mu_{0} g_{b} \sum_{l} C_{l} P_{l}^{1} \frac{r^{l}}{l+1},
\end{aligned}
$$

(note: $P_{l}^{1}=d P_{l}^{0} / d \theta$ )

In the bath $r>r_{2}$,

$$
\begin{aligned}
& V_{o}=\sum_{l} D_{l} r^{-(l+1)} P_{l}(\cos \theta), \\
& J_{\theta}=J_{o \theta}=-\frac{1}{r} g_{o} \frac{\partial V_{o}}{\partial \theta}=-g_{o} \sum_{l} r^{-(l+2)} D_{l} P_{l}^{1} .
\end{aligned}
$$

Since the total current integrated over all the space is zero, we deduce

$$
\begin{aligned}
I_{\text {enclosed }} & =-I_{\text {outside }}=\int_{0}^{2 \pi} \int_{r}^{\infty} J_{\theta} r \sin \theta d r d \phi \\
& =-g_{o} \sum_{l} D_{l} P_{l}^{1} 2 \pi \sin \theta \int_{r}^{\infty} r^{-(l+1)} d r \\
& =-\left.g_{o} \sum_{l} D_{l} P_{l}^{1} 2 \pi \sin \theta \frac{r^{-l}}{-l}\right|_{r} ^{\infty} \\
& =-g_{o} \sum_{l} D_{l} 2 \pi \sin \theta P_{l}^{1} \frac{r^{-l}}{l}, \\
B_{\phi} & =\frac{\mu_{0}}{2 \pi r \sin \theta} I_{\text {enclosed }}=-\mu_{0} g_{o} \sum_{l} D_{l} P_{l}^{1} \frac{r^{-(l+1)}}{l} .
\end{aligned}
$$

In the tissue $r_{1}<r<r_{2}$

$$
\begin{aligned}
V_{m} & =\sum_{l} F_{l} P_{l}(\cos \theta), \\
\psi & =\sum_{l}\left(A_{l} r^{\nu_{1}}+B_{l} r^{\nu_{2}}\right) P_{l}(\cos \theta), \\
J_{\theta} & =J_{i \theta}+J_{e \theta}=-\frac{1}{r} \frac{\partial\left(g_{i \theta} V_{i}+g_{e \theta} V_{e}\right)}{\partial \theta} \\
& =-\frac{1}{r} g_{i \theta} \frac{\partial\left(V_{i}+\left(g_{e \theta} / g_{i \theta}\right) V_{e}\right)}{\partial \theta}=-\frac{1}{r} g_{i \theta} \frac{\partial \psi}{\partial \theta} .
\end{aligned}
$$

The integral interval of (34) is split into two pieces: $0 \rightarrow r_{1}$ is $I_{1}$ and $r_{1} \rightarrow r$ is $I_{2}$.

$$
\begin{aligned}
I_{1} & =-\int_{0}^{2 \pi} \int_{0}^{r_{1}} J_{\theta} r \sin \theta d r d \phi \\
& =g_{b} \sum_{l} C_{l} P_{l}^{1} 2 \pi \sin \theta \frac{r_{1}^{l+1}}{l+1}, \\
I_{2} & =-\int_{0}^{2 \pi} \int_{r_{1}}^{r} J_{\theta} r \sin \theta d r d \varphi \\
& =g_{i \theta} 2 \pi \sin \theta \sum_{l} P_{l}^{1}\left\lfloor A_{l} \int_{r_{1}}^{r} r^{\nu_{1}} d r+B_{l} \int_{r_{1}}^{r} r^{\nu_{2}} d r\right\rfloor .
\end{aligned}
$$

The total enclosed current is then

$$
\begin{gathered}
I_{\text {enclosed }}=I_{1}+I_{2}=2 \pi \sin \theta P_{l}^{1} \sum_{l}\left\{g_{b} C_{l} \frac{r_{1}^{l+1}}{l+1}\right. \\
+g_{i \theta} A_{l} \frac{1}{v_{1}+1}\left(r_{2}{ }^{\nu_{1}+1}-r_{1}{ }^{\nu_{1}+1}\right)+g_{i \theta} B_{l} \\
\left.\cdot \frac{1}{v_{2}+1}\left(r_{2}{ }^{\nu_{2}+1}-r_{1}{ }^{\nu_{2}+1}\right)+g_{o} D_{l} \frac{r_{2}{ }^{-l}}{l}\right\} .
\end{gathered}
$$

Using (27) and (28), we get

$$
\begin{aligned}
& I_{\text {enclosed }} \\
& \quad=g_{i \theta} \sum_{l} P_{l}^{1} 2 \pi \sin \theta\left[\frac{A_{l}}{\nu_{1}+1} r^{\nu_{1}+1}+\frac{B_{l}}{v_{2}+1} r^{\nu_{2}+1}\right] \\
& B_{\phi}=\frac{\mu_{0}}{2 \pi r \sin \theta} I_{\text {enclosed }} \\
& \quad=\mu_{0} g_{i \theta} \sum_{l} P_{l}^{1}\left[\frac{A_{l}}{v_{1}+1} r^{\nu_{1}}+\frac{B_{l}}{v_{2}+1} r^{\nu_{2}}\right] .
\end{aligned}
$$

An advantage of this formulation is that by varying $r$ and $\theta$ the magnetic field is determined every where including within the heart wall.

\section{Results}

To perform calculations of the magnetic field, we need to consider a specific transmembrane potential distribution and values for the model parameters. We set the transmembrane 
potential to be $V_{m}=V_{2}=20 \mathrm{mV}$ for $\theta<89^{\circ}$ and $V_{m}=V_{1}$ $=-80 \mathrm{mV}$ for $\theta>91^{\circ}$. For the interval $89^{\circ}<\theta<91^{\circ}, V_{m}$ decreases linearly from 20 to $-80 \mathrm{mV}$. The heart size is $r_{1}$ $=30$ and $r_{2}=40 \mathrm{~mm}$ [10]. Furthermore, we set the blood and the bath conductivities to $g_{b}=g_{o}=1 \mathrm{~S} / \mathrm{m}$. The electrical conductivities of cardiac tissue are anisotropic: $g_{i r}=0.02, g_{i \theta}$ $=0.2, g_{e r}=0.08$, and $g_{e \theta}=0.2 \mathrm{~S} / \mathrm{m}[18]$.

Using the given transmembrane potential, we need to calculate its expansion in terms of Legendre polynomials.

3.1. The Calculation of Transmembrane Potential Expansion. To simplify the calculation, we change variables by letting $x=\cos \theta$. We let $x_{1}=\cos 91^{\circ}, x_{2}=\cos 89^{\circ}, \cos 0^{\circ}=1$, and $\cos 180^{\circ}=-1$, and then (24) becomes

$$
\begin{aligned}
F_{l} & =\frac{2 l+1}{2} \int_{-1}^{1} V_{m} P_{l}(x) d x \\
& =\int_{-1}^{x 1}+\int_{x 1}^{x 2}+\int_{x 2}^{1} V_{m} P_{l}(x) d x .
\end{aligned}
$$

For these integral intervals, (1) refers to $-1 \rightarrow x 1$, (2) refers to $x 1 \rightarrow x 2$, and (3) refers to $x 2 \rightarrow 1$. (1) is

$$
\int_{-1}^{x 1} V_{m} P_{l}(x) d x=V_{1} \int_{-1}^{x 1} P_{l}(x) d x .
$$

Using the equation of a line, $\left(V_{m}-V_{2}\right) /\left(x-x_{2}\right)=\left(V_{1}-\right.$ $\left.V_{2}\right) /\left(x_{1}-x_{2}\right)$, we get $V_{m}=V_{2}+\left(\left(V_{1}-V_{2}\right) /\left(x_{1}-x_{2}\right)\right)\left(x-x_{2}\right)$. Therefore, (2) is

$$
\begin{aligned}
& \int_{x 1}^{x 2} V_{m} P_{l}(x) d x \\
& =\int_{x 1}^{x 2}\left(V_{2}+\frac{V_{1}-V_{2}}{x_{1}-x_{2}}\left(x-x_{2}\right)\right) P_{l}(x) d x \\
& =V_{2} \int_{x 1}^{x 2} P_{l}(x) d x-x_{2} \frac{V_{1}-V_{2}}{x_{1}-x_{2}} \int_{x 1}^{x 2} P_{l}(x) d x \\
& \quad+\frac{V_{1}-V_{2}}{x_{1}-x_{2}} \int_{x 1}^{x 2} x P_{l}(x) d x .
\end{aligned}
$$

We need to obtain $\int P_{l}(x) d x$ and $\int x P_{l}(x) d x$ :

$$
\begin{aligned}
& \int_{x 1}^{x 2} x P_{l}(x) d x \\
& \quad=\frac{1}{2 n+1}\left[\left.\left(x P_{n+1}(x)-\int P_{n+1}(x) d x\right)\right|_{x 1} ^{x 2}\right. \\
& \left.\quad-\left.\left(x P_{n-1}(x)-\int P_{n-1}(x) d x\right)\right|_{x 1} ^{x 2}\right] .
\end{aligned}
$$

(3) is

$$
\int_{x 2}^{1} V_{2} P_{l}(x) d x=V_{2} \int_{x 2}^{1} P_{l}(x) d x .
$$

The final result is the sum of integrals of the three segments. Figure 2 is a comparison of the calculated approximate transmembrane potential with the original transmembrane potential, showing excellent agreement.

Figure 3 contains plots of the extracellular potential, calculated using (10), (16), and (17) with (19), (21), and (22). The potential falls off rapidly with distance into the bath or blood but is relatively large inside the cardiac tissue.

3.2. The Upper Bound of $l$ of the Approximated Sums. The magnetic field $\mathbf{B}$ is calculated in terms of Legendre polynomials. The infinite sums given above ( $l$ from zero to infinity) must be approximated as finite sums. Figure 4 shows the change of the calculated peak value of the magnetic field $\left(B_{\max }\right)$ as the total number of terms, $l$, in the sum increases. $B_{\text {peak }}$ changes by less than $3 \%$ for $l>200$, and therefore we use an upper bound $l=200$, which is enough to determine the magnitude of $\mathbf{B}$ accurately and is still easy to compute. When we choose $l=200$, the point with the maximum magnitude, $B_{\max }$, is about $14 \mathrm{nT}$.

Figure 5 displays the $\phi$-component of the magnetic field over a cross section of the heart. The field is largest near the inner and outer surfaces of the tissue and is relatively small throughout much of the heart wall. It has a peak magnitude, $B_{\max }$, of about 14 nT. Figure 6 shows the total current density (intracellular plus extracellular).

\section{Discussion}

The calculation of the magnetic field throughout the heart indicates that the peak magnetic field has a magnitude of about $14 \mathrm{nT}$. This is a similar order of magnitude as calculated by Roth [19] for a cylindrical strand of cardiac tissue, such as a papillary muscle, and is several times larger than the magnetic field at the apex of the heart (2 nT) [20,21], in planar tissue samples (1 nT) [22], or outside the body during measurements of the magnetocardiogram $(0.05 \mathrm{nT})$ [23]. The magnetic field is much larger at the tissue surface than just a few millimeters away from the surface, either in the surrounding bath or deeper in the tissue, which is again similar to the results found in a tissue strand [19]. This behavior arises because deep in the tissue the intracellular and extracellular currents are in the opposite directions with almost the same magnitude, and therefore they tend to cancel each other.

The calculation is based on a piecewise linear model for the transmembrane potential. The upstroke is distributed over $2^{\circ}$ in $\theta$, which is 0.035 radians. In the middle of the heart wall, the radius is $35 \mathrm{~mm}$, implying that the upstroke occurs over a distance of $1.22 \mathrm{~mm}$. This is a slightly slower upstroke than is typically observed in cardiac tissue. The rapid rise of the action potential occurs in about $1 \mathrm{~ms}$, and the conduction velocity is about $0.5 \mathrm{~m} / \mathrm{s}$ parallel to the fibers, implying an upstroke distributed over about $0.5 \mathrm{~mm}$. We performed a calculation with the upstroke over $1^{\circ}$ and found the peak magnitude of the magnetic field is about $16 \mathrm{nT}$, a $13 \%$ increase compared to $2^{\circ}$ case.

The cardiac tissue is assumed to have unequal anisotropy ratios $[18,24]$. However, this condition is not essential for producing the magnetic field. We repeated the calculation for equal anisotropy ratios, $g_{i r}=0.032, g_{i \theta}=0.2, g_{e r}=0.032$, and $g_{e \theta}=0.2 \mathrm{~S} / \mathrm{m}[18,24]$, and found that $B_{\max }$ changed only slightly, to $12 \mathrm{nT}$. 

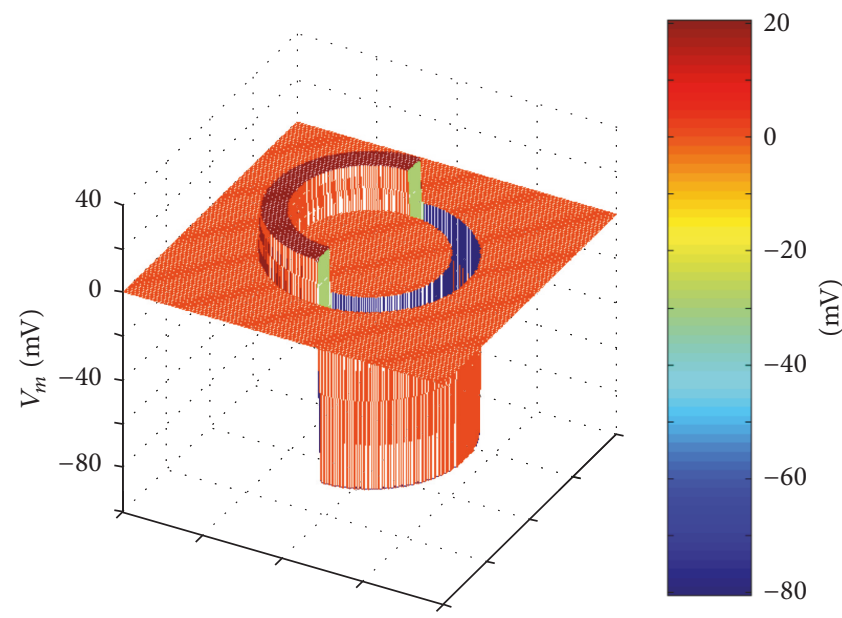

(a)
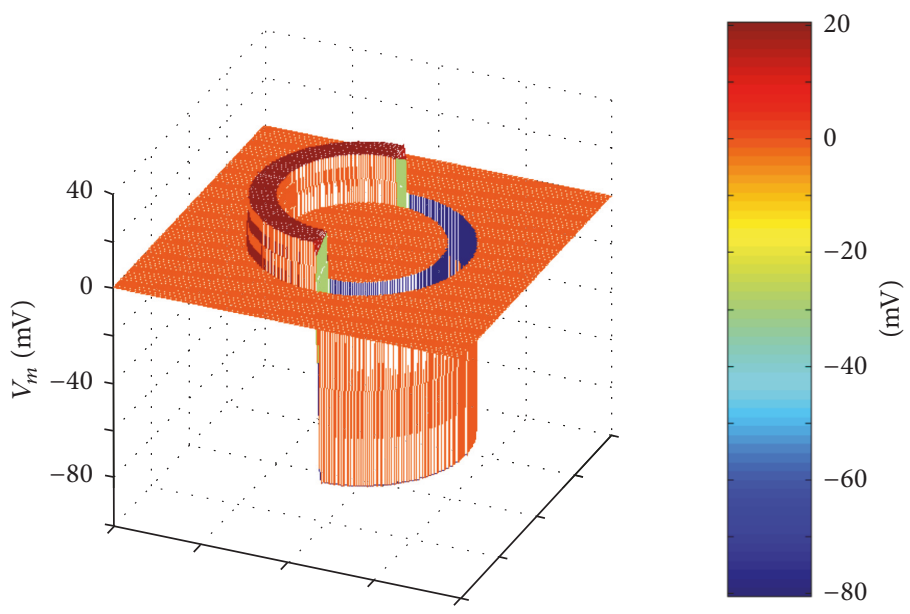

(b)

FIGURE 2: The transmembrane potential as a function of position over a cross section of the heart, (a) calculated for $l=200$, and (b) the specified transmembrane potential. The color bars are in $\mathrm{mV}$.
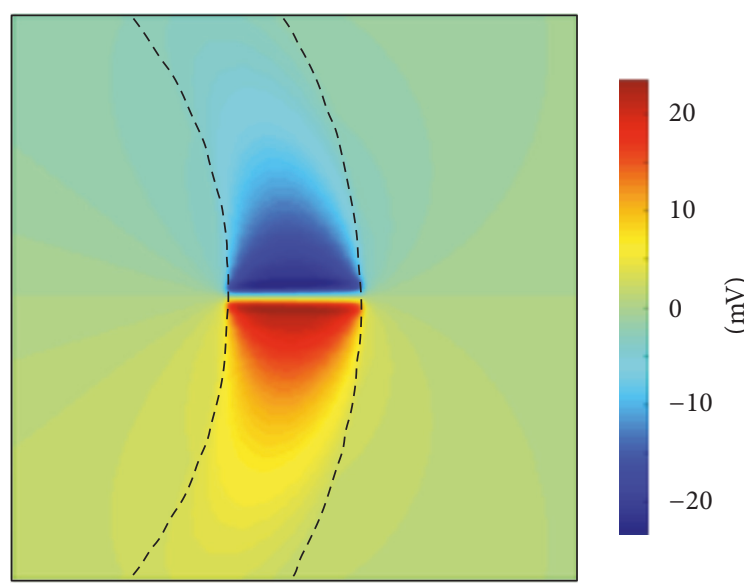

$-10$

$-20$

FIGURE 3: The extracellular potential over a cross section of the heart. The dashed curves indicate the heart inner and outer surfaces. An area $40 \mathrm{~mm}$ by $40 \mathrm{~mm}$ is shown.

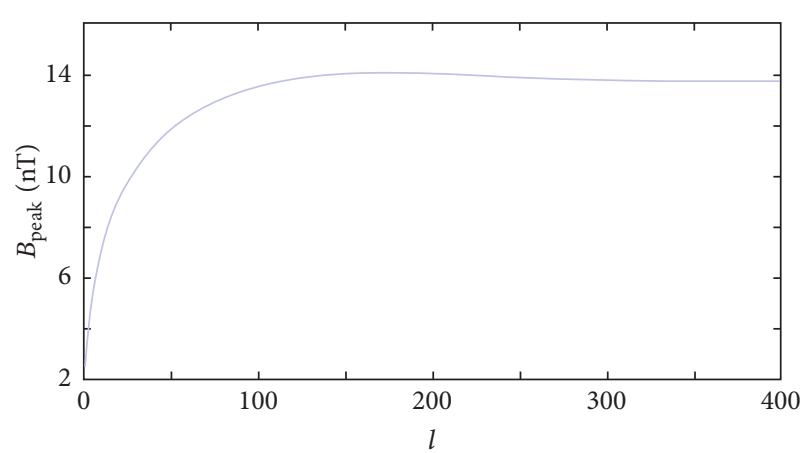

FIgURE 4: The peak magnetic field versus the maximum number of terms in the Legendre polynomial expansion, $l$.

Our calculation is based on a simplified model. The heart is spherical, the fiber geometry and wave front are idealized, and the transmembrane potential is uniform across the heart 


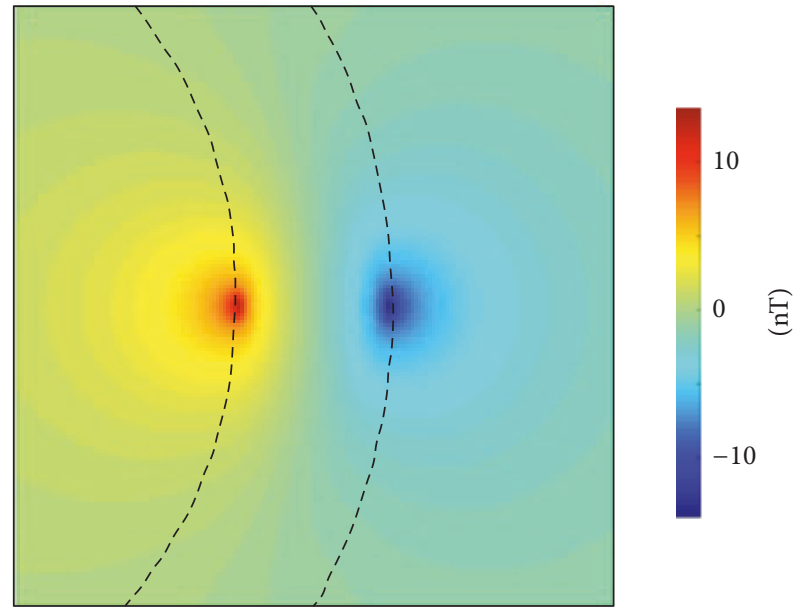

FIgURE 5: The magnetic field over a cross section of the heart. The dashed curves indicate the heart inner and outer surfaces. An area $40 \mathrm{~mm}$ by $40 \mathrm{~mm}$ is shown.

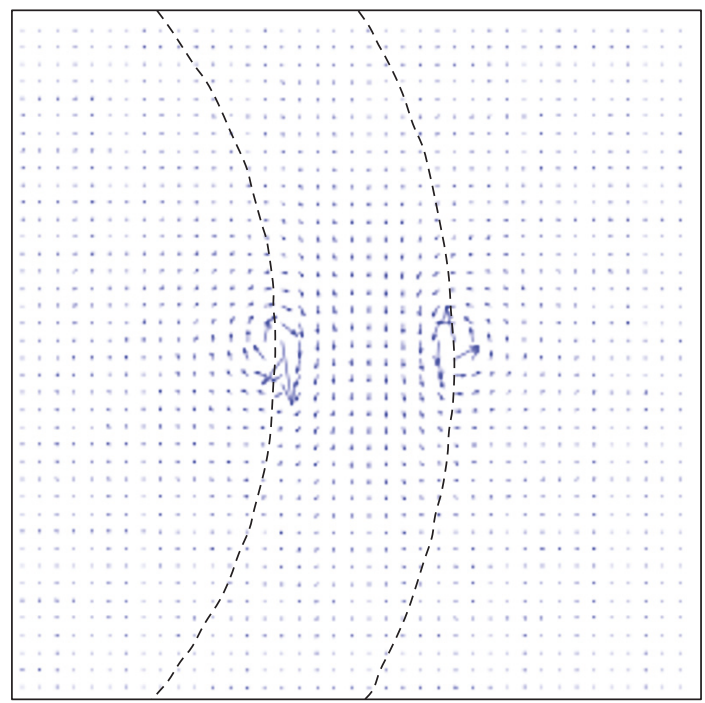

FIGURE 6: The current density (intracellular plus extracellular) over a cross section of the heart. The dashed curves indicate the heart inner and outer surfaces. An area $40 \mathrm{~mm}$ by $40 \mathrm{~mm}$ is shown.

wall. These assumptions are not physiologically accurate but are necessary in order to calculate the biomagnetic field analytically. More realistic calculations would require numerical methods.

4.1. How Action Currents Affect MRI. The groundbreaking work of Joy and his colleagues demonstrated that MRI can be used to measure current by detecting its resulting magnetic field [25-27]. However, much of this work has examined applied current, such as during defibrillation [28]. Detecting biocurrents is a greater challenge, because they are so small.

In living neural or cardiac tissues there exist various physiological processes such as blood flow, diffusion, and chemical exchange. All these physiological processes alter the magnetic field surrounding the atomic nuclei in ways that affect the magnetic resonance behavior of the nuclei [29]. Therefore, special MRI acquisition methods were designed or need to be designed accordingly to observe different physiological functions.

Functional magnetic resonance imaging (fMRI) makes use of the Blood Oxygenation Level Dependent (BOLD) signal changes [30]. Briefly, deoxyhemoglobin in blood is paramagnetic which will distort the external magnetic field of nearby hydrogen nuclei spins and further affect the MR image. When part of brain is active, the vascular system provides more blood and thereby reduces the fraction of blood that is converted from oxyhemoglobin into deoxyhemoglobin, and the magnetic distortion decreases. BOLD fMRI provides a method to measure oxygenation perfusion and thereby to indirectly estimate brain activity. However the spatial and temporal distribution of perfusion may differ from the distribution of electrical activity. Therefore, researchers would like to measure electrical activity directly using MRI.

Can the magnetic field produced by bioelectric currents be used as the gradient field for MRI? Theoretically, it is possible. The question is if the resulting phase shift is large enough to detect by current MRI systems. The phase shift $\Phi$ of the MRI signal is given approximately by [5]

$$
\Phi=\gamma B \Delta t,
$$

where $\gamma=267 \times 10^{6}$ radians/(s T) is the gyromagnetic ratio for protons and $\Delta t$ is the time over which the phase accumulates (the duration of the magnetic field for brief biomagnetic signals or a duration related to the MRI pulse sequence such as the echo time for long duration biomagnetic signals).

The phase shift determines if the electrical activity can be detected by the MRI system. In the brain, although the phase shift produced by a single neuron firing is not detectable, neural firings are not isolated events; in fact they are correlated with each other. There are two ways to increase the MR signal so that it is detectable. One is doing spatial and temporal integrals of neuronal firings over an MRI voxel according to the morphologies and the physiology of the neuronal events; the other one is altering the acquisition method such as using special pulse sequences to increase phase shift. Many researchers have been doing this both experimentally and theoretically $[1-8,31-34]$. The MRI signals they measure are very small, and it is still unclear if they are detectable, whether the results are "fantasy, possibility, or reality" [3]. Recently, Sundaram et al. [5] performed neural current imaging in an intact turtle cerebellum using MRI. They were able to detect biomagnetic fields of strength of up to one nanotesla and phase shifts of about a third of a degree. These measurements required signal averaging with thousands of repetitions, imaged long duration metabotropic-induced neural signals lasting nearly a second, and had no interference caused by the vascular response (BOLD signal), tissue motion related to pulsatile blood flow, or other sources of physiological noise. Under these idealized conditions, phase shifts on the order of a third of a degree are detectable. 
4.2. Phase Shifts by Action Currents in the Heart. The brain is not the source of the largest biocurrents in the body; the heart is, so it may be easier to detect cardiac action currents using MRI compared to neural action currents. Deep in the heart, the magnetic field is small since the intracellular and the extracellular currents have almost the same value but are in opposite directions. However near the surface of the heart the extracellular current leaves the heart with the "return" loops through an adjacent volume conductor which produces a significant magnetic field [11].

From this analysis, we have achieved our original goal of computing the maximum value of the magnetic field produced by the action currents near the surface of the heart, $14 \mathrm{nT}$, which is significantly larger than any magnetic field produced by neuronal activation. For a firing time of $1 \mathrm{~ms}$, this magnetic field can produce a maximum phase shift of $0.21^{\circ}$ by (47). This phase shift is similar to that measured by Sundaram et al. [5], implying that the cardiac signal may be just measurable using MRI. However, these signals may be smaller than artifacts caused by heart motion and blood flow effects in a beating heart. Therefore, in vivo measurements of cardiac biocurrents in humans remain a challenge. Perhaps more promising would be to search for such signals in a Langendorff-perfused heart with an electromechanical uncoupler to eliminate contractions. In such an in vitro system-somewhat analogous to the in vitro turtle cerebellum model used by Sundaram et al. [5] to study signals from the brain-biocurrents in the heart may be measureable. Our conclusion is that the magnetic field in the heart may be detectable under idealized conditions but that using MRI to measure action currents in the humans will be difficult.

4.3. The Potential of Detecting Cardiac Arrhythmias Using MRI. "Arrhythmia" is a term describing conditions in which the heart is beating too fast (tachycardia), too slow (bradycardia), or irregularly. Abnormal electrical activity exists in the heart in each of these conditions.

The usual way to detect a cardiac arrhythmia is using an electrocardiogram (ECG). Magnetic fields are also sometimes measured (the magnetocardiogram [23], or MCG). In MCG measurements, the biomagnetic field is measured outside the body and a difficult inverse problem must be solved to obtain the current sources that produce the magnetic field [35]. Simplifying assumptions are required to make the inverse problem solvable and stable, but these assumptions make the resulting current source distribution less precise and detailed. MRI would provide a way to measure the magnetic field inside the heart directly, avoiding the inverse problem of deducing the field inside the heart from measurements far outside the heart. Much research has been done that attempts to measure the magnetic field of a nerve using MRI. However, the heart, which produces a much larger magnetic field than nerves, may be a better place to start when attempting to use MRI for measurement of biocurrents. Of course, many challenges exist, not the least of which is the motion of the beating heart and the differences between the paramagnetic susceptibility of the blood within the heart, the cardiac tissue, and the tissues outside the heart $[36,37]$.
However, if successful, obtaining biocurrents from an MRI of the heart would provide a closer look at the function of the heart's electrical conduction system and should help to diagnose a large number of cardiac arrhythmias.

\section{Conflicts of Interest}

The authors declare that they have no conflicts of interest.

\section{Acknowledgments}

This research was supported by the National Institutes of Health, Grant R01EB008421.

\section{References}

[1] H. Kamei, K. Iramina, K. Yoshifcawa, and S. Ueno, "Neuronal current distribution imaging using magnetic resonance," IEEE Transactions on Magnetics, vol. 35, no. 5, pp. 4109-4111, 1999.

[2] J. Bodurka and P. A. Bandettini, "Toward direct mapping of neuronal activity: MRI detection of ultraweak, transient magnetic field changes," Magnetic Resonance in Medicine, vol. 47, no. 6, pp. 1052-1058, 2002.

[3] P. A. Bandettini, N. Petridou, and J. Bodurka, "Direct detection of neuronal activity with MRI: Fantasy, possibility, or reality?" Applied Magnetic Resonance, vol. 29, no. 1, pp. 65-88, 2005.

[4] A. M. Cassarà, G. E. Hagberg, M. Bianciardi, M. Migliore, and B. Maraviglia, "Realistic simulations of neuronal activity: a contribution to the debate on direct detection of neuronal currents by MRI," NeuroImage, vol. 39, no. 1, pp. 87-106, 2008.

[5] P. Sundaram, A. Nummenmaa, W. Wells et al., "Direct neural current imaging in an intact cerebellum with magnetic resonance imaging," NeuroImage, vol. 132, pp. 477-490, 2016.

[6] W. I. Jay, R. S. Wijesinghe, B. D. Dolasinski, and B. J. Roth, "Is it possible to detect dendrite currents using presently available magnetic resonance imaging techniques?" Medical and Biological Engineering and Computing, vol. 50, no. 7, pp. 651-657, 2012.

[7] J. Huang, "Detecting neuronal currents with MRI: a human study," Magnetic Resonance in Medicine, vol. 71, no. 2, pp. 756$762,2014$.

[8] G. E. Hagberg, M. Bianciardi, and B. Maraviglia, "Challenges for detection of neuronal currents by MRI," Magnetic Resonance Imaging, vol. 24, no. 4, pp. 483-493, 2006.

[9] D. Xu and B. J. Roth, "The magnetic field produced by the heart and its influence on MRI," in Proceedings of SIAM Conference, Great Lakes Section, Dearborn, Mich, USA, April, 2010.

[10] N. A. Trayanova, B. J. Roth, and L. J. Maiden, "The Response of a spherical heart to a uniform electric field: a bidomain analysis of cardiac stimulation," IEEE Transactions on Biomedical Engineering, vol. 40, no. 9, pp. 899-908, 1993.

[11] R. A. Murdick and B. J. Roth, "A comparative model of two mechanisms from which a magnetic field arises in the heart," Journal of Applied Physics, vol. 95, no. 9, pp. 5116-5122, 2004.

[12] C. S. Henriquez, "Simulating the electrical behavior of cardiac tissue using the bidomain model," Critical Reviews in Biomedical Engineering, vol. 21, pp. 1-77, 1993.

[13] J. C. Neu and W. Krassowska, "Homogenization of syncytial tissues," Critical Reviews in Biomedical Engineering, vol. 21, pp. 137-199, 1993.

[14] B. J. Roth and J. P. Wikswo, "A bidomain model for the extracellular potential and magnetic field of cardiac tissue," IEEE 
Transactions on Biomedical Engineering, vol. 33, no. 4, pp. 467469, 1986.

[15] B. J. Roth, "A comparison of two boundary conditions used with the bidomain model of cardiac tissue," Annals of Biomedical Engineering, vol. 19, no. 6, pp. 669-678, 1991.

[16] W. Krassowska and J. C. Neu, "Effective boundary conditions for syncytial tissues," IEEE Transactions on Biomedical Engineering, vol. 41, no. 2, pp. 143-150, 1994.

[17] M. Abramowitz and I. A. Stegun, Handbook of Mathematical Functions: with Formulas, Graphs and Mathematical Tables, Dover Publications, New York, NY, USA, 1965.

[18] B. J. Roth, "Electrical conductivity values used with the bidomain model of cardiac tissue," IEEE Transactions on Biomedical Engineering, vol. 44, no. 4, pp. 326-328, 1997.

[19] B. J. Roth, Longitudinal Resistance in Strands of Cardiac Muscle [Ph.D. thesis], Vanderbilt University, Nashville, Tenn, USA, 1987.

[20] B. J. Roth, W.-Q. Guo, and J. Wikswo, "The effects of spiral anisotropy on the electric potential and the magnetic field at the apex of the heart," Mathematical Biosciences, vol. 88, no. 2, pp. 191-221, 1988.

[21] K. K. McBride, B. J. Roth, V. Y. Sidorov, J. P. Wikswo, and F. J. Baudenbacher, "Measurements of transmembrane potential and magnetic field at the apex of the heart," Biophysical Journal, vol. 99, no. 10, pp. 3113-3118, 2010.

[22] J. R. Holzer, L. E. Fong, V. Y. Sidorov, J. P. Wikswo Jr., and F. Baudenbacher, "High resolution magnetic images of planar wave fronts reveal bidomain properties of cardiac tissue," Biophysical Journal, vol. 87, no. 6, pp. 4326-4332, 2004.

[23] H. Koch, "Recent advances in magnetocardiography," Journal of Electrocardiology, vol. 37, pp. 117-122, 2004.

[24] B. J. Roth, "How the anisotropy of the intracellular and extracellular conductivities influences stimulation of cardiac muscle," Journal of Mathematical Biology, vol. 30, no. 6, pp. 633-646, 1992.

[25] M. Joy, G. Scott, and M. Henkelman, "In vivo detection of applied electric currents by magnetic resonance imaging," Magnetic Resonance Imaging, vol. 7, no. 1, pp. 89-94, 1989.

[26] G. C. Scott, M. L. G. Joy, R. L. Armstrong, and R. M. Henkelman, "Sensitivity of magnetic-resonance current-density imaging," Journal of Magnetic Resonance, vol. 97, no. 2, pp. 235-254, 1992.

[27] G. C. Scott, M. L. G. Joy, R. L. Armstrong, and R. M. Henkelman, "Measurement of nonuniform current density by magnetic resonance," IEEE Transactions on Medical Imaging, vol. 10, no. 3, pp. 362-374, 1991.

[28] R. S. Yoon, T. P. DeMonte, K. F. Hasanov, D. B. Jorgenson, and M. L. G. Joy, "Measurement of thoracic current flow in pigs for the study of defibrillation and cardioversion," IEEE Transactions on Biomedical Engineering, vol. 50, no. 10, pp. 1167-1173, 2003.

[29] R. K. Hobbie and B. J. Roth, Intermediate Physics for Medicine and Biology, Springer, New York, NY, USA, 5 edition, 2015.

[30] S. Ogawa, T. Lee, A. S. Nayak, and P. Glynn, "Oxygenation-sensitive contrast in magnetic resonance image of rodent brain at high magnetic fields," Magnetic Resonance in Medicine, vol. 14, no. 1, pp. 68-78, 1990.

[31] R. H. Kraus Jr., P. Volegov, A. Matlachov, and M. Espy, "Toward direct neural current imaging by resonant mechanisms at ultralow field," NeuroImage, vol. 39, no. 1, pp. 310-317, 2008.

[32] A. M. Cassarà and B. Maraviglia, "Microscopic investigation of the resonant mechanism for the implementation of nc-MRI at ultra-low field MRI," NeuroImage, vol. 41, no. 4, pp. 1228-1241, 2008.
[33] M. N. J. Paley, L. S. Chow, E. H. Whitby, and G. G. Cook, "Modelling of axonal fields in the optic nerve for direct MR detection studies," Image and Vision Computing, vol. 27, no. 4, pp. 331-341, 2009.

[34] R. S. Wijesinghe and B. J. Roth, "Detection of peripheral nerve and skeletal muscle action currents using magnetic resonance imaging," Annals of Biomedical Engineering, vol. 37, no. 11, pp. 2402-2406, 2009.

[35] E. A. Lima, A. Irimia, and J. P. Wikswo, "The magnetic inverse problem," in The SQUID Handbook, J. Clarke and A. I. Braginski, Eds., vol. 2, Applications, pp. 139-267, Wiley-VCH, Weinheim, Germany, 2006.

[36] J. P. Wikswo, "Noninvasive magnetic detection of cardiac mechanical activity: Theory," Medical Physics, vol. 7, no. 4, pp. 297-306, 1980.

[37] J. P. Wikswo, J. E. Opfer, and W. M. Fairbank, "Noninvasive magnetic detection of cardiac mechanical activity: experiments," Medical Physics, vol. 7, no. 4, pp. 307-314, 1980. 


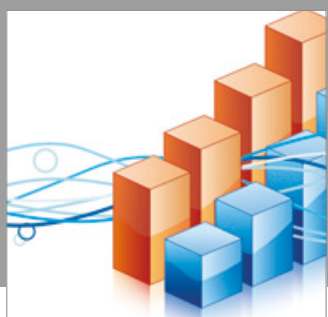

Advances in

Operations Research

vatersals

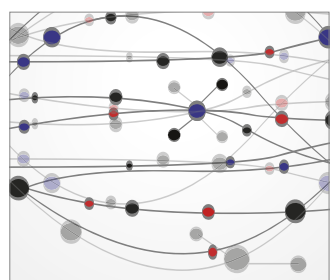

\section{The Scientific} World Journal
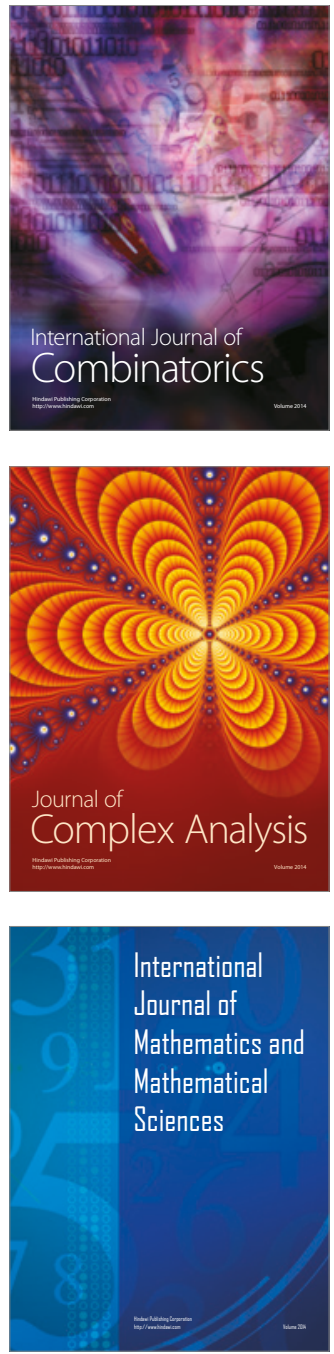
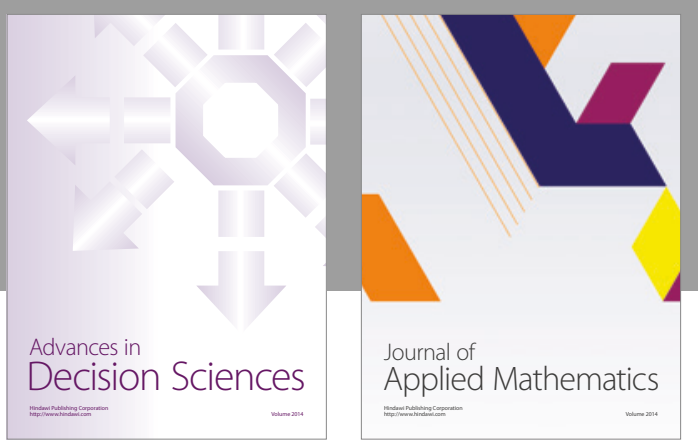

Algebra

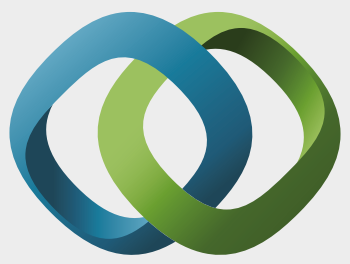

\section{Hindawi}

Submit your manuscripts at

https://www.hindawi.com
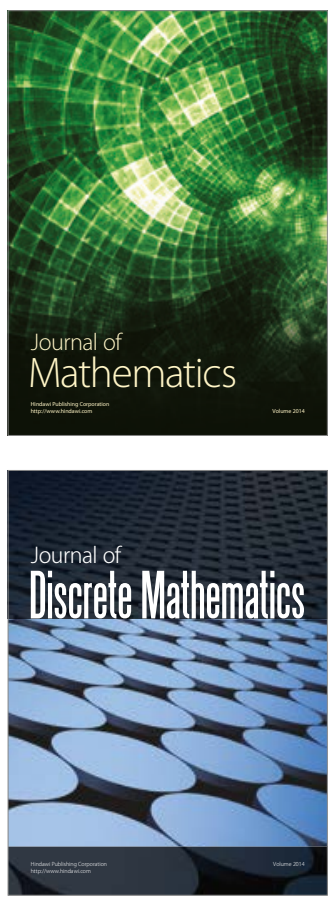

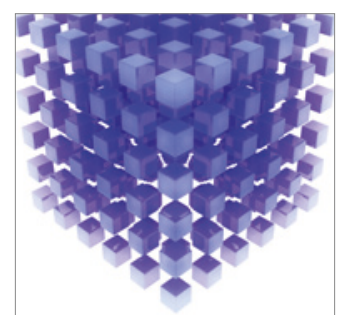

Mathematical Problems in Engineering
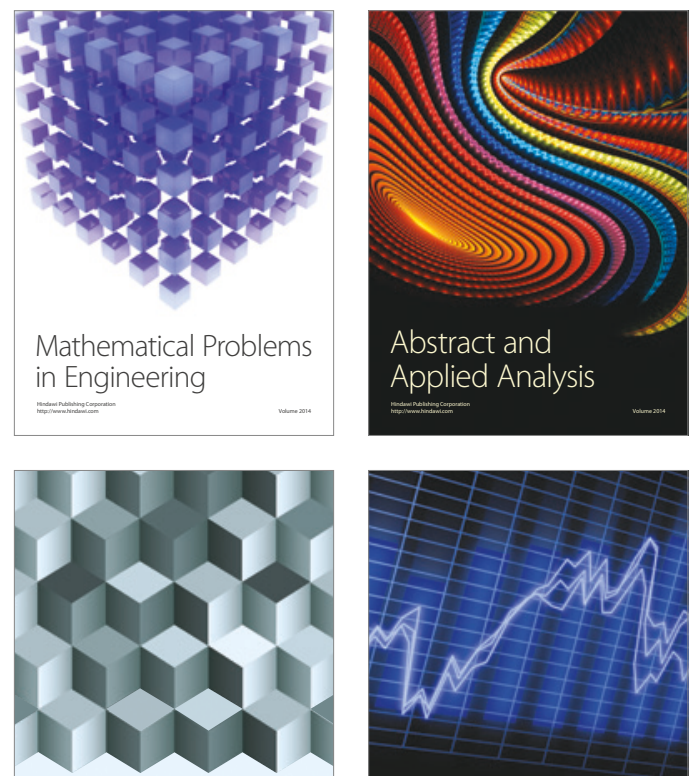

Journal of

Function Spaces

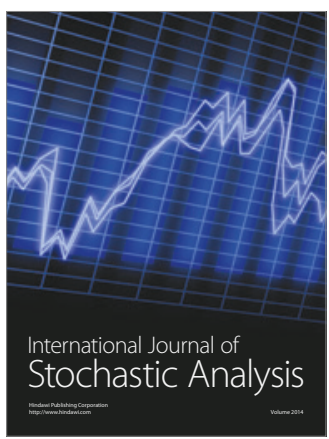

Probability and Statistics
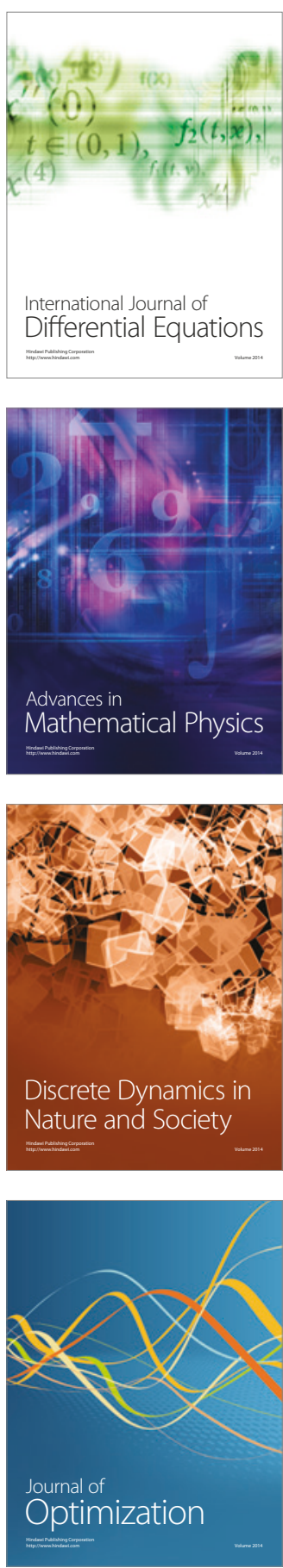